Revue d'histoire de l'Amérique française

DE REVUE D.HISTOIRE DE L'AMÉRIQUE FRANÇAISE

\title{
Le projet d'histoire sociale de la population du Saguenay : l'appareil méthodologique
}

\section{Gérard Bouchard et Yolande Savoie}

Volume 32, numéro 1, juin 1978

URI : https://id.erudit.org/iderudit/303672ar

DOI : https://doi.org/10.7202/303672ar

Aller au sommaire du numéro

Éditeur(s)

Institut d'histoire de l'Amérique française

ISSN

0035-2357 (imprimé)

1492-1383 (numérique)

Découvrir la revue

Citer cet article

Bouchard, G. \& Savoie, Y. (1978). Le projet d'histoire sociale de la population du Saguenay : l'appareil méthodologique. Revue d'histoire de l'Amérique française, 32(1), 41-56. https://doi.org/10.7202/303672ar d'utilisation que vous pouvez consulter en ligne. 


\title{
LE PROJET D'HISTOIRE SOCIALE DE LA POPULATION DU SAGUENAY: L'APPAREIL MÉTHODOLOGIQUE
}

\author{
GÉRARD BOUCHARD \\ Département des Sciences bumaines \\ Université du Québec à Chicoutimi \\ YOLANDE LAVOIE \\ Statistique Canada
}

Nous achevons dans les pages qui suivent la présentation générale du Projet d'Histoire sociale de la Population du Saguenay (1842-1941), commencée dans deux articles antérieurs où il a été fait largement état des objectifs scientifiques et des orientations théoriques de cette recherche ${ }^{1}$. Nous nous proposons ici de décrire, tout en les justifiant au besoin, les instruments et les méthodes adoptées, de même que les multiples directions d'analyses qu'ils ouvrent à l'histoire sociale.

Prévenons d'abord le lecteur que ce projet a lui-même une histoire, c'est-à-dire qu'il a connu en cours de route des tâtonnements inévitables et quelques volte-faces qui ont impliqué des ajustements parfois substantiels; on se gardera donc de penser que la

1 Voir Gérard BOUCHARD, «L'histoire de la population et l'étude de la mobilité sociale au Saguenay, $\mathrm{XIX}^{e} \cdot \mathrm{XX}^{\mathrm{e}}$ siècles», Recherches sociographiques, XVII, 3 (sept.-déc. 1976): 353-372; Gérard BOUCHARD, «Introduction à l'étude de la société saguenayenne aux $\mathrm{XIX}^{\mathrm{e}}$ et $\mathrm{XX}^{\mathrm{e}}$ siècles», Revue d'histoire de l'Amérique française, 31, 1 (juin 1977): 3-27. Rappelons que ce projet de recherche bénéficie depuis 1973 de l'assistance financière du Conseil des Arts du Canada et que l'équipe de recherche se compose, outre les auteurs du présent article, de Christian Pouyez et Raymond Roy, du département des Sciences humaines de l'Université du Québec à Chicoutimi, de Patrick Brard et François Martin, du Centre d'Informatique de l'UQAC et de Michel Bergeron, recherchiste pour le projet depuis cinq années. D'autre part, précisons que jusqu'à l'automne 1977 , notre recherche avait pour cadre chronologique la période 1842-1931; des dispositions prises récemment ont permis d'étendre ce cadre jusqu'à l'année 1941 .

RHAF, vol. 32, $\mathrm{n}^{\circ} 1$, juin 1978 
cohésion de nos démarches a été spontanément obtenue. La technologie qui sert d'appui à l'histoire sociale (ce qu'il est convenu d'appeler, d'un terme trop vague, les techniques quantitatives) a évolué avec une grande rapidité depuis dix ans. Ainsi tous les projets de recherche qui sont nés durant cette période et qui ont fait une large place à l'ordinateur ont été victimes, à des degrés divers, d'une évolution avec laquelle ils ont dû négocier des compromis successifs. Nous n'y avons pas échappé. Tel de nos procédés, encore neuf il y a cinq ans, paraîtra aujourd'hui désuet, entendons: trop lent et trop coûteux. Nous espérons toutefois démontrer que l'essentiel - c'est-à-dire la précision et la cohésion de la démarche scientifique - est sauf.

L'idée de cette enquête est née en 1970 à l'occasion d'une recherche sur Laterrière, village saguenayen situé à une dizaine de milles au sud de Chicoutimi. Notre projet initial était de faire de cette paroisse une sorte de Crulai québécois. Tous les registres de baptêmes, mariages et sépultures ont été dépouillés pour la période 1855-1970, à partir de quoi quelques milliers de dossiers de familles ont été manuellement constitués. Les premières analyses devaient cependant révéler l'ampleur des mouvements migratoires dont cette population était l'objet, la majorité des dossiers devenant ainsi inutilisables pour l'étude de la fécondité et de la mortalité, notamment ${ }^{2}$. En même temps que d'autres, nous découvrions la vulnérabilité des monographies de type Crulai lorsque expérimentées sur des populations instables.

Parallèlement à cette recherche villageoise, nous avions amorcé une première tentative d'enquête régionale dont le but était de reconstituer par ordinateur les familles d'une douzaine de paroisses saguenayennes pour la période allant de 1842 , date d'ouverture des premiers registres, à 1970 . Projet qui, lui aussi et pour les mêmes

2 Il en a été tiré, comme on imagine, quelques leçons de méthode; voir à ce sujet Gérard Bouchard, «L'histoire démographique et le problème des migrations: l'exemple de Laterrière », Histoire sociale / Social History, III, 15 (mai 1975): 21-33. Les dossiers de famille de Laterrière ont néanmoins été exploités et ils ont fourni quelques mesures utiles de l'évolution démographique villageoise (structure de la population, migrations, intervalles, nuptialité, etc.). Ces résultats ont été présentés au Congrès de la Société historique du Canada à Kingston en 1973. Dans l'ensemble, nous n'avons jamais regretté ce pas de clerc qui nous a permis de constituer une première banque de dossiers de familles saguenayennes lesquels nous ont constamment servi depuis, à la façon d'une population-laboratoire, pour la mise au point de notre méthodologie. 
raisons, a dû être abandonné ${ }^{3}$. Ces premiers essais ont suggéré d'autres amendements qui ont finalement donné à notre projet sa forme actuelle: une enquête d'histoire sociale de la population du Saguenay (incluant le Lac St-Jean) de 1842 à 1941, ayant pour but de recréer l'évolution d'ensemble d'une société régionale. Ce dernier trait est fondamental. Dans l'étape initiale, il est vrai, nous nous sommes employés à mettre au point une infrastructure de recherche, faite d'instruments et de procédés (reconstitution automatique des familles, jumelage de dossiers) qui seront appliqués en un premier temps à un corpus de données majoritairement démographiques, et il sera longuement question de cela dans l'exposé qui va suivre. Mais ces priorités sont imposées par un échéancier et elles ne sauraient masquer l'objectif général poursuivi, lequel commandera en son temps des tâches plus familières à l'historien et où le social retrouvera pleinement ses droits.

\section{LES SOURCES}

Il a semblé utile de nous interroger d'abord sur la population: dénombrer les hommes, fixer le rythme de leur reproduction, suivre leur répartition dans l'espace et leurs déplacements. Les premiers dépouillements ont ainsi porté sur les registres de baptêmes, mariages, sépultures et sur les recensements nominatifs, tout cela représentant une masse imposante à recueillir. Pour la période 1842-1941, les registres de l'ensemble des paroisses du Saguenay (une vingtaine en 1900 , près de 60 pour 1941) contiennent 340,000 actes, dont près des deux tiers ont été dépouillés à ce jour. La série que nous utilisons est celle de la partie civile et elle est déposée au greffe des Palais de justice de Chicoutimi et de Roberval ${ }^{4}$. Il n'y a pas de registres manquants et l'état matériel des documents est excellent. Nous avons

3 Non sans porter quelque fruit cependant, sous la forme cette fois d'un échantillon représentatif de villages saguenayens dûment calibré à partir d'indices socio-économiques très divers et rigoureusement compilés; cf. Gérard BOUCHARD, «Histoire démographique et histoire quantitative. A propos d'un échantillon de paroisses rurales au Saguenay ", Protée, 3, 2 (printemps 1974): 17-28.

${ }_{4}$ Depuis le $17^{\mathrm{e}}$ siècle en France (ordonnance de Saint-Germain-en-Laye, 1667) et au Québec (éđit du Conseil souverain, 1678), les registres de baptêmes, mariages et sépultures sont tenus en double, une série étant conservée dans les paroisses, l'autre dans les greffes civils. Voir sur cette question Gérard Bouchard et André Larose, "La réglementation du contenu des actes de baptême, mariage et sépulture au Québec, des origines à nos jours", Revue d'histoire de l'Amérique française, 30, 1 (juin 1976): 67-84. 
en outre terminé le dépouillement des manuscrits des recensements réalisés en 1852, 1861 et 1871 par les soins du gouvernement canadien. Les recensements nominatifs effectués par les curés lors de leur visite paroissiale font présentement l'objet d'un dépouillement exhaustif qui devrait être achevé dans un an; cette série est cependant fort incomplète et la plupart des documents conservés portent sur le $20^{\mathrm{e}}$ siècle. Diverses listes nominatives ont également été mises à profit: recensements d'arpenteur, listes de souscripteurs, collections d'histoires de vie.

Au total, plus de 200000 fiches de relevé ont été remplies depuis le début de ce projet, lesquelles donneront lieu prochainement à un premier travail d'analyse. L'ensemble des dépouillements prévus sera terminé d'ici deux ou trois ans. Toutefois, l'exploitation systématique et approfondie de la banque de données devra être précédée d'une critique sévère du fichier destinée à l'expurger des erreurs qui s'y glissent au cours des opérations de saisie des données (cf. infra). Peùtêtré ce geñre de tráváail ne trouve-t-il pas toujours ia place qui lui revient dans les enquêtes d'histoire et de sciences sociales. Il est pourtant indispensable dans la mesure où il assure d'une manière incontestable la rationalité des procédés et la précision des résultats. Dans cette direction, quelques étapes ont déjà été franchies ${ }^{5}$. Mais il s'agit surtout d'études de sources préliminaires; les travaux à venir porteront directement sur la critique de la banque de données à l'aide de l'ordinateur. Ces opérations se dérouleront en quatre temps:

a) Examen des séries chronologiques (en vue d'établir la fidélité du double du greffe par rapport à celui des paroisses) et analyse descriptive du contenu des actes de baptême, mariage et sépulture, par paroisse et par décennie.

b) Critique de l'enregistrement des naissances, mariages et sépultures à l'aide des dossiers de familles reconstituées.

5 Voir Gérard Bouchard et André Larose, «La réglementation...» (article cité); «Sur l'enregistrement civil et religieux au Québec du XVII ${ }^{\mathrm{e}}$ au $\mathrm{XX}^{\mathrm{e}}$ siècle», à paraître au début de 1978 , in A. Côté, Répertoire d'archives régionales, Ministère des Affaires culturelles, Québec; Gérard Bouchard et Michel Bergeron, «Les registres de l'état civil de Notre-Dame-de-Laterrière, 1855-1911», Archives 75.3, III, 3 (sept.décembre 1975): 164-173; «L'arpenteur J.-B. Duberger et les premiers recensements de la population saguenayenne au XIX ${ }^{\mathrm{e}}$ siècle», Archives, 8, 3 (décembre 1976): 11-20. Pour ce qui concerne l'étude des registres, signalons ici l'apport considérable qu'a représenté pour nous un fonds généalogique saguenayen couvrant les années 1842-1870 et qui nous a été généreusement donné par MM. Jean-Paul Simard et Léonidas Larouche. 
c) Évaluation critique des fiches de familles à l'aide des recensements nominatifs disponibles.

d) Correction du fichier et révision du cadre d'analyse ${ }^{6}$.

D'autres problèmes sont soulevés par la nécessité de fondre des données puisées à des sources différentes. Un exemple récent nous en a été fourni par la mise au point des instruments en vue de l'étude des mouvements migratoires à l'échelle de la famille et de l'individu. Cette étude s'appuiera sur les recensements nominatifs fédéraux et sur les registres paroissiaux, par le truchement des dossiers de familles reconstituées. Mais il advient que: 1) l'unité spatiale de base des recensements est le canton ou la municipalité, tandis que celle des registres est la paroisse religieuse; 2) d'une décennie ou d'une année à l'autre, ces unités font l'objet de constants réaménagements, les divisions de recensement faisant $\mathrm{fi}$ des frontières municipales ou cantonales et les circonscriptions religieuses se défaisant sans cesse au gré de l'ouverture des nouvelles paroisses. Ces problèmes d'équivalence ont été récemment surmontés grâce à un long travail de reconstitution cartographique qui nous a conduits à créer des nouvelles divisions territoriales. Ces nouvelles divisions effacent les divergences entre les unités premières et assurent un jumelage parfait des données des recensements et des registres ${ }^{7}$.

Mentionnons enfin, au chapitre des sources, les rapports annuels des curés à leur évêque. Les renseignements démographiques contenus dans ces rapports (population des paroisses, migrations...) ont été entièrement relevés pour la période $1842-1972$ et ils serviront d'appui à une étude préliminaire sur le mouvement général de la population saguenayenne: marche du peuplement, urbanisation, taux de natalité, nuptialité, mortalité, structure de la population, mobilité géographique.

\section{LA SAISIE DES DONNÉES}

Nous nous proposons de décrire sous ce titre les instruments à l'aide desquels l'ensemble des données ont été recueillies et mises sur bandes magnétiques; il sera fait état, pour les registres et pour les recensements des opérations relatives à la cueillette, au codage, à

6 Cette partie de la recherche est sous la responsabilité de M. Raymond Roy.

Cf. Christian Pouyez et Michel Bergeron, "L'étude des migrations au Saguenay (1842-1931): problèmes de méthode». A paraître au printemps 1978 dans Histoire sociale/Social History. 
la vérification, au stockage des données par ordinateur et aux tests de validation automatique. Il n'est cependant pas possible dans le cadre de cet article de fournir des explications complètes et certains points essentiels ne seront qu'évoqués ${ }^{8}$.

\section{A - Les registres de baptêmes, mariages, sépultures}

Nous nous en sommes remis, pour le dépouillement des registres au procédé conventionnel qui consiste à transcrire le contenu des actes sur feuilles de relevé. Ce procédé a pour caractéristique d'être très sûr et très lent. Des essais récents ont été faits ici et là en vue d'accélérer la saisie des données. Il convient de signaler sous ce rapport la formule qui a été mise au point par des chercheurs (MM. Couturier, A. Chamoux, J. Dupâquier) du Laboratoire de Démographie historique de l'École des Hautes Études en Sciences sociales de Paris. Cette formule consiste à relever au magnétophone le contenu des actes et elle permettrait d'atteindre des vitesses insoupçonnées au dépouillement (de l'ordre de 150 actes à l'heure). L'évaluation qualitative de ce procédé ne semble pas avoir été poussée à fond, en termes de marges d'erreurs notamment; mais chacun reconnaît le progrès auquel il peut ouvrir l'accès, à condition de s'appuyer sur des règles très rigoureuses.

\section{a) Les fiches de relevé}

Nos trois fiches de relevé (une pour chaque type d'acte) ont été mises au point en 1972, à partir du modèle classique proposé par Louis Henry. Le contenu des actes y est transcrit soit en clair, soit sous forme codée. Nous avons été particulièrement attentifs à effectuer un relevé définitif qui doit épargner tout travail de dépouillement aux utilisateurs de ces données.

b) Le codage

Sur ce point, la prudence nous a conduits à construire des codes extrêmement décomposés et très rigides qui ne laissent guère d'initiative aux releveurs et qui garantissent une transcription fidèle

8 Les lecteurs désireux d'obtenir des informations complémentaires sont invités à contacter les auteurs de cet article. Nous tenons aussi à la disposition des intéressés des exemplaires de tous les instruments de recherche (fiches de relevé, manuels de codage, grilles de contrôle, etc.) et des 27 documents de travail qui ont été produits dans le cadre du Projet d'Histoire sociale de la Population du Saguenay. 
du contenu des actes. Pour ce qui concerne les données socioprofessionnelles par exemple, nous nous sommes gardés d'un procédé hâtif qui aurait consisté à établir un classement à priori dont l'analyse aurait été ensuite captive. Plutôt, nous nous sommes contentés d'assigner simplement à chaque profession un numéro qui est une sorte de matricule, remettant à plus tard le soin d'effectuer par ordinateur toutes les formules souhaitables de classement ou de hiérarchisation ${ }^{9}$.

Nous pensons que cette façon de procéder assortie de quelques dispositions qui seront décrites plus bas, nous ont permis d'éviter les principaux pièges inhérents à la cueillette et au codage des données, tels: l'absence d'uniformité dans le travail de codage, la difficulté de transcrire intégralement et fidèlement le contenu des documents, l'impossibilité d'évaluer avec une précision suffisante les résultats de ces opérations ${ }^{10}$.

\section{c) Les instruments de contrôle}

Nous nous sommes attachés tout particulièrement à suivre la marche du dépouillement, dans le but de contrôler à la fois le rythme du travail et les erreurs commises. À cette fin, nous avons mis au point un système de vérification qui opère par le truchement de 9 grilles de contrôle. Ces instruments font ressortir pour chaque releveur les vitesses horaires et le genre de fautes commises (d'omission, de distraction, d'interprétation) selon le type d'acte et les rubriques affectées (mentions nominatives, dates, etc.).

Depuis le début du travail, $10 \%$ des fiches compilées ont fait l'objet d'une vérification intégrale. Nous avons pu suivre ainsi de très près l'évolution des vitesses horaires et des \% d'erreurs, et nous ménager une possibilité immédiate d'intervention. Les résultats

9 L'exemple à suivre, en ce domaine difficile du traitement des données socio-professionnelles, est sans contredit celui du Philadelphia Social History Project qui a élaboré à cette fin un corpus d'instruments et de procédés extrêmement souples; voir le numéro spécial de Historical Methods Newsletter, 9, 2-3 (mars-juin 1976), en particulier Théodore Hershberg et Robert Dockhorn, "Occupational Classification»: 59-98. Voir aussi T. Hershberg, M. Katz, S. Blumin, L. Glasco et Clyde Griffen, "Occupation and ethnicity in five nineteenth century cities: A collaborative inquiry», Historical Methods Newsletter, VII, 3 (juin 1974): 174-216.

10 Les problèmes ont été exposés avec force récemment; voir Lawrence STONE, "History and the Social Sciences in the Twentieth Century», in Charles F. Delzell (éd.), The Future of History (Nashville, Vanderbilt University Press, 1977), 34. 
des contrôles ont prouvé l'efficacité de ces procédés. Mentionnons par exemple que la quantité d'erreurs a été diminuée de moitié durant les trois premières années du dépouillement ${ }^{11}$, le rythme du dépouillement se situant à 15 actes/heure.

\section{d) Le stockage et la validation automatique des données}

La mise des données sur bandes magnétiques s'effectue directement à l'aide d'un terminal et en interactif, par ordinateur XEROX 560. Les actes ainsi traités (au nombre de 175,000 jusqu'ici) sont disponibles en tout temps pour correction ou listage. Mais auparavant, ils sont l'objet d'une validation automatique destinée à dépister les erreurs commises, non plus seulement par les releveurs, mais aussi par les déclarants, les rédacteurs des actes et les opérateurs du terminal. Cette validation consiste en une série de tests destinés à détecter des erreurs ayant échappé aux contrôles manuels qui suivent immédiatement le dépouillement. Enfin, deux autres types de validation interviendront après que les famililes auront été reconstituées par ordinateur, l'une prenant la forme de tests de cohérence appliqués aux dossiers de famille, l'autre consistant en une confrontation de ces mêmes dossiers avec les fiches de ménages issues des recensements nominatifs. Au terme de ces opérations, nous estimons que le fichier sera entaché d'une quantité d'erreurs négligeable, soit une erreur pour 60 actes environ ${ }^{12}$.

\section{$B$ - Les recensements nominatifs}

Parallèlement au dépouillement des registres, nous nous sommes attaqués à diverses listes nominatives, dont les principales sont les recensements du gouvernement canadien et ceux des paroisses ${ }^{13}$.

11 La marche des opérations de contrôle, l'application des grilles, la série des résultats et les indices d'erreurs utilisés sont exposés en détail dans nos documents de travail nos 5 et 27.

12 Cela représente une performance légèrement supérieure à celle réalisée par J. Sutter qui a travaillé sur de très petites quantités ( 5000 fiches) se prêtant aisément à une vérification exhaustive. Voir Annales de Démographie historique, (1966): 62-63, 77 (rapport d'une discussion à une séance régulière de la Société). Mais il est difficile d'aller très loin dans la voie de la comparaison. Jusqu'ici il semble que les chercheurs ont attaché une importance très inégale au contrôle du dépouillement, un grand nombre se limitant à des vérifications partielles dont les résultats ne sont pas toujours connus.

13 Parmi les autres, mentionnons les trois recensements effectués par l'arpenteur Duberger au début de la colonie; voir sur ce sujet Gérard Bouchard et Michel Bergeron, "L'arpenteur J.-B. Duberger et les premiers recensements de la population saguenayenne au XIX ${ }^{\mathrm{e}}$ siècle», Archives, 8,3 (décembre 1976): 11-20. 
Les premiers sont disponibles pour les années $1852,1861,1871$ et ils portent sur l'ensemble des paroisses existantes à ces années-là (soit 5,12 et 16 respectivement). Ils ont été entièrement dépouillés. Les seconds sont des recensements annuels opérés par les curés; plus de soixante ont été dépouillés à ce jour.

\section{a) Le dépouillement}

Pour toutes ces listes nominatives, une fiche de relevé a été dessinée qui reprend une partie des rubriques contenues dans les fiches de dépouillement des registres; mais il s'en ajoute une foule d'autres, issues pour la plupart des recensements canadiens, toujours plus riches que les dénombrements paroissiaux. Nous avons été attentifs à observer ici les mêmes règles qui ont été exposées plus haut: transcription intégrale et fidèle du contenu des recensements, uniformité du codage (à l'aide d'un deuxième manuel rédigé à cette fin), évaluation du dépouillement.

Nous nous sommes autorisés, suivant en cela quelques précédents accrédités et à partir d'indices fournis par les documents eux-mêmes, à inférer au moment du dépouillement les relations de parenté entre les membres du ménage - étant donné que les recenseurs, avant 1881, ne donnaient pas cette information. Cependant, pour éviter les risques inhérents à cette opération, nous avons pris un certain nombre de précautions, parmi lesquelles la construction d'un code d'incertitudes ${ }^{14}$, et nous avons veillé à ce que ces décisions ne soient irréversibles, nous ménageant ainsi la faculté de rejeter ces inférences si elles se révélaient mal fondées ${ }^{15}$.

14 A l'image de celui qui a été utilisé par Barbara Laslett dans ses études sur les familles de Los Angeles; voir, entre autres, "Social change and the family: Los Angeles, California, 1850-1870», American Sociological Review, 42, 2 (avril 1977): 272-274.

15 Pour des raisons qui tiennent surtout à l'état des recensements paroissiaux, nous avons jusqu'ici repoussé l'idée d'effectuer par ordinateur ce travail de déduction. Une équipe qui est allée très loin dans cette dernière direction est celle du Philadelphia Social History Project. Voir Theodore HERSHBERG, «A method for the computerized study of family and household structure using the manuscript schedule of the U.S. Census of population, 1850-1880", The Family in Historical Perspective, 3 (printemps 1973): 5-20. Aussi, Buffington C. Miller, A computerized Method of Determining Family Structure from Mid-Nineteenth Century Census Data, Ph. D. (Université de la Pennsylvanie, 1972). Ces auteurs ont travaillé sur les recensements américains, dont les recensements canadiens sont une réplique assez fidèle. 
b) Les étapes de la validation et le stockage des données

Afin de garantir l'application uniforme des règles ci-haut mentionnées, les fiches de relevé ont fait l'objet d'une vérification continuelle en cours de travail. La nature des difficultés posées par les recensements interdisait toutefois le genre de contrôles mis au point pour le dépouillement des registres de même qu'une quantification très poussée de l'évaluation. Aussi, un test effectué sur les recensements canadiens a démontré qu'il n'était pas utile de prolonger davantage nos efforts dans cette direction; par exemple sur la transcription des données nominatives, le codage du sexe, de l'état matrimonial, des lieux de résidence, etc., on retrouvait un pourcentage d'erreurs très bas, sans doute attribuable aux lenteurs dont s'est entouré le dépouillement de ces recensements ${ }^{16}$.

C'est donc sur la validation automatique des données que nous avons concentré nos efforts. Les tests mis au point à cette fin ressemblent à ceux qui ont été construits pour les registres. Mais ils donnent un rendement supérieur car le grand nombre de rubriques contenues dans les recensements fédéraux permet de détecter plus facilement les données contradictoires ${ }^{17}$.

\section{PROCÉDÉS DE TRAITEMENT ET DIRECTIONS D'ANALYSE}

Après le jumelage par ordinateur des dossiers de familles et des recensements fédéraux - tâche qui sera réalisée d'ici trois ans au maximum - nous disposerons d'un fichier socio-démographique d'une grande richesse qui fera l'objet d'une première exploitation, dans la direction maintenant classique de la démographie historique - avec la différence que nous sommes assurés de contrôler dans une large mesure le phénomène de la mobilité démographique ${ }^{18}$.

16 Ce qui vient d'être dit sur la méthode de dépouillement des recensements mériterait certainement de plus amples justifications. Elles feront bientôt l'objet d'une présentation appropriée.

17 Voir Premiers tests de validation automatique des données des recensements nominatifs, Document de travail no 12 (1976), 15 pages. Nous remercions l'équipe du P.R.D.H., du département de démographie de l'Université de Montréal, qui a bien voulu nous transmettre une copie de ses propres tests, sur le modèle desquels nous avons construit les nôtres.

18 Il n'est pas utile d'insister longuement sur l'importance capitale de ce dernier point; chacun sait comment la mobilité géographique des populations peut perturber les fichiers des chercheurs, mettant parfois en échec les plans d'analyse les mieux établis. 
L'étape qui sera abordée ensuite ouvrira la porte à une histoire sociale assez inédite, dont il convient de dire quelques mots.

Nous prévoyons en effet achever, d'ici un an ou deux, la mise au point de nos programmes de reconstitution automatique des familles ${ }^{19}$. Nous fixerons ensuite les modalités du jumelage registresrecensements (travail qui est déjà en cours), d'où il résultera une banque d'individus et de familles ayant séjourné au Saguenay depuis 1842. Après l'analyse démographique, les données du fichier seront réaménagées de manière à passer des dossiers de famille aux fiches individuelles. Dans cette opération, les dossiers de familles ne seront pas démantelés, mais les éléments les plus stables de leur contenu formeront une sorte de matricule accolé à chacun des membres. Par exemple, le matricule d'un individu $\mathrm{X}$ pourrait comprendre les initiales des prénoms et noms de ses parents, la date de leur mariage, la date et le lieu (codé) de naissance de X. Cette disposition, qui préserverait la référence aux dossiers de familles et aux ménages, donnerait un accès direct et sûr aux fiches biographiques. En outre, elle préparerait le terrain à la cueillette de nouvelles informations relatives, cette fois, aux individus. Nous allons en effet intégrer à la banque de données ainsi constituée des renseignements à caractère économique, social, culturel, médical, etc. Ces informations viendront s'ajouter au contenu des fiches et transformeront la banque de données en un fichier individuel universel et automatisé. Techniquement, nous avons déjà pris quelques dispositions essentielles qui nous garantissent la possiblité d'ajouter à volonté des informations sur les fiches personnelles, lesquelles deviendront en quelque sorte des dossiers cumulatifs. Certes, nous n'espérons pas qu'un tel fichier sera absolument complet puisqu'il y manquera à la fois des personnes et des renseignements importants. Nous nous attendons toutefois à ce qu'il ouvre l'accès à une grande variété d'analyses et à ce que, pour chacune des questions qui feront l'objet d'enquêtes, il soit possible de constituer des échantillons très largement représentatifs.

En ce moment, nous planifions l'exploitation de nos données selon quatre grandes directions de recherche:

a) Fondements physiques et biologiques

- Épidémiologie

- Génétique (notamment: étude des maladies héréditaires par la construction automatique des arbres généalogiques)

19 Nous nous apprêtons à rendre public les résultats d'un test effectué sur un échantillon de près de 2000 actes tirés des registres de la paroisse de Laterrière. 
Anthropométrie

b) Comportements démographiques

- Fécondité, nuptialité, mortalité

- Mouvements migratoires à l'échelle régionale

- Genèse et structure de la population (étudiée par le biais de recensements automatiques)

- Etc.

c) Structures sociales

- Hiérarchies socio-économiques

- Clivages et rapports sociaux (ex.: analyse des choix conjugaux)

- Étude de la mobilité sociale entre générations

- Élites socio-politiques (mécanismes, rythmes de reproduction, de renouvellement)

- Groupe familial

d) Culture

- Criminalité

- Alphabétisation

- Accès à l'enseignement collégial, universitaire; choix de carrières

- Participation à la vie religieuse: analyse des vocations religieuses, du personnel des associations pieuses et des diverses œuvres paroissiales.

Cette liste n'est pas exhaustive; elle peut s'allonger au gré des sources et des fichiers déjà disponibles ou à constituer. Parmi les premiers, mentionnons: un fichier nominatif intégral des personnes, hommes et femmes, nées au Saguenay et devenues membres du clergé séculier ou régulier depuis l'origine du diocèse jusqu'à 1945; un fichier nominatif de tous les étudiants ayant été inscrits au Petit Séminaire de Chicoutimi de 1873 à 1948; un fichier nominatif comprenant 400 dossiers d'histoire socio-professionnelle et familiale. L'ensemble de ces fiches individuelles, il va sans dire, contient toutes les indications suffisantes (date et lieu de naissance, nom et prénoms des parents, etc.) pour effectuer aisément leur jumelage avec le fichier central. D'autre part, il y a nombre d'autres fichiers à créer à partir de sources existantes, d'excellente qualité, comme les rôles de l'évaluation foncière, les sources de la propriété immobilière, les archives judiciaires, les dossiers de candidatures électorales, de patients, de pèlerins, les listes de souscripteurs, d'abonnés, de pétitionnaires, d'actionnaires, etc.

Ces conceptions ne sont pas entièrement nouvelles. Il convient d'abord de rappeler qu'elles sont appuyées sur une ambition qui est 
au cœur d'une très vieille tradition historiographique, savoir l'idée de l'histoire totale. Il s'y ajoute, bien sûr, des éléments techniques et méthodologiques essentiels qui ont eux aussi des précurseurs, du côté de l'administration hospitalière par exemple, ou de la médecine ${ }^{20}$. Dans les sciences sociales, l'idée a été esquissée dès 1966 par M. Couturier, lors d'une séance de la Société de démographie historique ${ }^{21}$, par Roger Schofield à Léningrad en $1970^{22}$, par Jacques Dupâquier en $1973^{23}$, ensuite par Mark H. Skolnick ${ }^{24}$. Elle est à la base de l'entreprise amorcée depuis dix ans par le P.R.D.H. à l'Université de Montréal et elle a d'ores et déjà inspiré des recherches tout à fait novatrices à partir des recensements américains et canadiens du $19^{\circ}$ siècle $^{25}$. Cependant, toutes les tentatives de jumelage ou de reconstitution automatique faites jusqu'à ce jour ont été handicapées à des degrés divers soit par les difficultés (financières) d'accès à l'ordinateur, soit par la qualité des sources - registres de baptêmes, mariages, sépultures et recensements nominatifs. De ce point de vue, le Projet d'Histoire sociale de la Population du Saguenay réunit des conditions qui lui confèrent un avantage incontestable, notamment parce que la recherche porte sur une période récente. Nous serons sans doute en mesure, par exemple, de reconstituer automatiquement les familles avec un rendement et un degré de précision jamais atteints jusqu'ici ${ }^{26}$.

20 Voir E.D. Acheson, Record Linkage in Medexine (Londres, E.S. Livingstone, 1968), 399 pages (actes d'un colloque tenu à Oxford en juillet 1967). Voir en particulier l'exposé de H. B. Newcombe, pp. 7-33.

21 «... rassembler sur le nom du même homme tous les renseignements qui concernent cet homme». Cf. Annales de Démographie historique (1966): 69 et 70 .

22 Cf. «La reconstitution de la famille par ordinateur», Annales E.S.C., (juillet-octobre 1972), 4-5: 1071-1082.

23 Voir, entre autres, "Ombres et lumières en démographie historique», dans la revue Dix-huitième siècle (1973), 5: 59-65. L'auteur y évoque, au passage, les recherches qu'il a entreprises sur le Vexin français «avec l'ambition d'étudier et de mesurer les rapports qui peuvent exister à l'échelle individuelle entre comportements démographiques, niveaux de fortune, professions, mobilité géographique, participation politique, etc.» (p. 61).

24 The Construction and Analysis of Genealogies from Parish Registers with a Case Study of Parma Valley, Italy. Thèse de doctorat (Université Stanford, 1974), 265 pages (cf. p. 6).

25 Mentionnons, parmi quelques autres, le Philadelphia Social History Project (directeur: Theodore Hershberg), le Canadian Social History Project (directeur: Michael Katz), le Peel County History Project (directeur: David Gagan).

${ }^{26}$ Cet énoncé s'appuie sur les résultats du test évoqué plus haut et qui seront bientôt publiés. 
La banque de données ainsi constituée, même appuyée sur des techniques de traitement extrêmement souples, efficaces, et si polyvalente qu'elle puisse être, n'épuise évidemment pas le champ de l'histoire sociale. Il lui manque l'appoint indispensable des données dites qualitatives. De même, les amas de matériaux qu'on en tirera ne vaudront que dans la mesure où ils s'appuieront sur des hypothèses et des cadres théoriques clairement établis ${ }^{27}$. Ces remarques sont faites dans le but de nous éviter, si possible, d'inutiles et coûteux procès de «quantitativisme» ou d'on ne sait quel positivisme. L'intérêt et l'opportunité d'entreprises comme la nôtre procèdent simplement d'un souci de rigueur et de l'avantage qu'il y a, de ce point de vue, à appliquer à l'enquête historique les techniques modernes de traitement des données. Sur la plupart des sentiers pratiqués par l'histoire sociale, ces fichiers cumulatifs promettent de jeter un éclairage qui renouvelle et approfondisse le questionnement scientifique. À propos de très vieilles interrogations rattachées à la mobilité sociale, à la reproduction des élites, à l'évolution concrète des rapports sociaux considérés dans la longue période et pour l'ensemble d'une population, les enquêtes à venir ne manqueront pas de produire quelques belles pièces à conviction ${ }^{28}$. Dans d'autres directions aussi, moins familières à l'historien mais combien prometteuses et pressantes, il faudra engager des ressources en grande quantité: c'est bien sûr, l'histoire sociale du corps, des maladies, de la mort; mais c'est aussi l'étude de la génétique, maintenant au seuil de progrès spectaculaires grâce à la possibilité de construire automatiquement les arbres généalogiques ${ }^{29}$. De ce point de vue, les perspectives sont exaltantes puisque nous envisageons de prolonger nos dépouillements en deçà de 1842 , dans la direction de Charlevoix et de réaliser à long terme la jonction entre notre fichier et celui de l'équipe Charbonneau-Légaré (P.R.D.H.). Toutes ces possibilités concrètes,

27 Les nôtres ont été exposés dans un article déjà cité, cf. Gérard Bouchard, «Introduction à...", Revue d'histoire de l'Amérique française, 31, 3 (juin 1977): 3-27.

28 Cf. Gérard Bouchard, «L'histoire de la population et l'étude de la mobilité sociale», article cité. À noter que ce qu'on désigne du nom de "prosopographie» s'apparente étroitement avec cette veine de recherche (voir L. Stone, «Prosopography», Daedalus (hiver 1971): 46-79); avec cette différence que, appuyée sur un fichier universel de la population, l'enquête prosopographique pourra enfin, et comme le souhaite Lawrence Stone, déborder le fief des élites au profit de clientèles populaires.

29 C'est encore, en retour, la faculté de valider les chaînes de familles reconstituées à l'aide de contrôles hémotypologiques... 
déjà en cours de réalisation, autorisent les plus grands espoirs pour les progrès de la connaissance du passé et du présent.

\section{UNE HISTOIRE À PART ENTIÈRE}

Ce titre emprunté à Lucien Febvre traduit très fidèlement le sens de nos travaux. Le Projet d'Histoire sociale de la Population du Saguenay a pour objet de: 1) dépouiller d'une manière exhaustive et systématique les grandes sources nominatives de l'histoire sociale, dans le but de constituer, pour une population régionale, un fichier biographique cumulatif étalé dans la longue période, 2) assujettir ces données à des programmes de traitement automatique, 3) plier l'analyse historique à des interrogations multiples, souvent novatrices, et parfois suivies - pourquoi pas? - de réponses. A cette fin, nous avons voulu conjuguer quelques innovations majeures survenues dans la science historique depuis l'époque où Louis Henry fixait le modèle de la monographie villageoise de démographie historique. Ce sont les suivantes:

a) L'usage intensif de l'ordinateur pour le jumelage des données, en particulier pour la reconstitution des familles.

b) Le passage de l'échelle villageoise à l'échelle régionale.

c) Le dépouillement et le jumelage de tous les recensements nominatifs disponibles.

d) L'intégration massive de données non démographiques aux fiches individuelles et familiales, d'où la nécessité de problématiques et d'équipes interdisciplinaires.

e) L'exploitation de liaisons inter-familles pour l'étude des diverses formes et mécanismes de transmission (sociale aussi bien que biologique ou culturelle).

f) Le prolongement de l'enquête historique jusqu'aux temps présents.

Ainsi constitués, il n'est pas illusoire d'espérer que de tels fichiers pourront fournir à l'histoire sociale les fondements méthodologiques et techniques dont elle a besoin pour concrétiser pleinement sa vocation interdisciplinaire. Par sa nature même, le jumelage automatique des données est un procédé irrespectueux des frontières et autres formes de cloisonnements. Sur le plan technique, il est peut-être une contre-partie de toutes les entreprises théoriques qui, depuis le $\mathrm{XIX}^{\mathrm{e}}$ siècle, ont voulu procurer aux sciences sociales une vision unifiée de leur objet. La perspective de marier ces deux 
trajectoires représente un défi qui vaut d'être relevé, quelles qu'en soient les incertitudes.

Et au-delà de cette conjecture, il demeure la possibilité d'édifier une infrastructure durable pour la connaissance historique de telle sorte que, pour tout ce qui touche aux couches les plus dures, les mieux objectivables de l'objet social, celle-ci acquerrait certains éléments de permanence, sinon d'accumulation. Entendons-nous bien cependant; nous ne pensons pas ici à une accumulation intégrale et linéaire du savoir historique, fondée sur la croyance naive en une histoire objective et définitive ${ }^{30}$.

Plutôt, notre énoncé évoque la possibilité et la nécessité de rationaliser et d'uniformiser de larges parties de l'enquête historique qui ne ressortissent pas vraiment au travail d'interprétation où la subjectivité conserve évidemment ses droits. Telles sont les tâches de cueillette et d'élaboration des données; telles sont certaines compilations élémentaires qui, pour peu qu'elles soient effectuées rigoureusement, sont entièrement récupérables, quelles que soient les hypothèses de la recherche; tels encore une foule de programmes et d'instruments d'analyse à reconnaitre soigneusement au cours de concertations à venir.

Tous ces éléments seront conjugués dans le fichier que nous construisons et dont les données sont assujetties à trois conditions fondamentales: 1) l'exhaustivité, 2) l'accessibilité universelle, 3) la polyvalence des analyses et des interrogations. Ces trois règles garantissent une neutralité maximale et sont de nature à épargner aux usagers éventuels le soin de retourner à quelque source documentaire ayant servi à la constitution de la banque.

Ces outils sont évidemment coûteux, de même que les instruments de traitement dont ils doivent être assortis: mais l'histoire traditionnelle l'est-elle donc moins, dont les procédés et les contraintes portent si souvent à la hâte, à la querelle et à de continuels recommencements à nouveaux frais?

30 Conception qui, il est vrai, se retrouve chez certains adeptes de l'histoire quantitative; voir par exemple Lee Benson, Toward the Scientific Study of History. Selected Papers (Philadelphia, M. B. Lippincott Co., 1972). 